\title{
Convergence Analysis for Generalized Ant Colony Optimization Algorithm
}

\author{
Daiyuan Zhang \\ ${ }^{1}$ College of Computer, Nanjing University of Posts and Telecommunications, Nanjing \\ 210003 \\ ${ }^{2}$ Institute of Computer Technology, Nanjing University of Posts and Telecommunications, \\ Nanjing 210003
}

\begin{abstract}
A new algorithm is proposed, which is called Generalized Ant Colony Optimization (GACO) algorithm. Two new functions are presented to model the behavior for describing the pheromone evaporation and pheromone added to the edges that belong to the best-so-far solution. A class of strictly increasing function is proposed, which gives a general form of expression for the probability of selecting the next node. An important theorem is proved for describing the convergence of GACO algorithm, i.e. for a sufficiently large number of algorithm iterations, the probability of finding the globally optimal solution at least once tends to 1 .
\end{abstract}

Keywords: artificial intelligence, ant colony optimization, ant algorithms, convergence proof, approximation algorithms, GACO algorithm.

\section{Introduction}

In ant colony optimization (ACO) algorithms, some artificial ants are introduced to construct solutions to the considered problem from the indirect communication mediated by the environment [1]. The construction of good solutions is a result of the artificial ants' cooperative interaction.

Although a number of applications to many different-hard combinatorial opti- mization problems [2] [3] has empirically shown the effectiveness of ant colony optimization, scientists have developed very little theory to explain the reasons underlying ACO's success.

At present, scientists know litter about the behavior of real ants, which implies that it is very difficult to simulate the behavior of real ants by artificial methods. Therefore, we have to introduce artificial ants for simplifying our analysis.

Although there may be some differences between the behavior of artificial ants and the behavior of real ants in the world, our purpose is, through learning the complex behavior of animals, to find some effective methods for solving some difficult problems.

For example, Birattari et al. [4] have proposed an interpretation of ACO in the framework of optimal control and reinforcement learning. Meuleau and Dorigo [5] have shown that ACO algorithms and stochastic gradient descent are strongly related and that a particular form of ACO algorithms converges to a local optimum with probability 1 . Gutjahr proved convergence to the globally optimal solution with probability $1-\varepsilon$ of a particular ant colony optimization algorithm called graph-based ant system (GBAS) [6]. Stützle and Dorigo presented a simple convergence proof for $\mathrm{ACO}_{\mathrm{gb}, \tau_{\min }}$ algorithm [7], where gb indicates that the global best pheromone update rule is used,

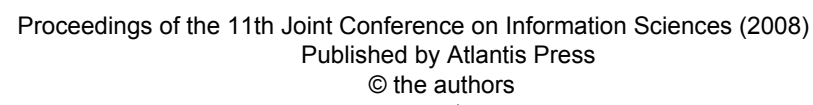


and $\tau_{\min }$ indicates that a lower limit on the range of feasible pheromone trails is enforced.

Artificial ants (or ants for short in the following) are also introduced in GACO algorithm. Different from $\mathrm{ACO}_{\mathrm{gb}, \tau_{\text {min }}}$ algorithm[7], a general formula for ant solution construction is proposed in GACO algorithm, while the formula for ant solution construction presented in $\mathrm{ACO}_{\mathrm{gb}, \tau_{\text {min }}}$ algorithm is a particular form of GACO algorithm. A new feasible pheromone trails update method is also introduced for the global best pheromone update rule. A proposition is proved for describing the upper bound and the lower bound of the pheromone trails. An important theorem is proved for describing the convergence of GACO algorithm.

\section{Generalized Ant Colony Optimiza- tion Algorithm}

Artificial ants can build candidate solutions by performing randomized walks on the completely connected, weighted graph for the considered problem. Each artificial ant is put on a randomly chosen node (vertex) of the graph and then it performs a randomized walk by moving at each step from the source node to the target node in the graph in such a way that the next node (vertex) is chosen stochastically according to the strength of the pheromone currently on the edges (arcs). While moving from one node to another of the graph, constraints are used to prevent ants from building infeasible solutions. Once the ants have completed their walk, pheromone trails are updated. The general form of expression for the probability selecting the next node by an ant at a source node is described as follows.

ANT SOLUTION CONSTRUCTION of GACO ALGORITHM
While (the next vertex be-
longs to the components of
the problem) do:
At iteration m, for each
source node $i$, select the
next node (or target node)
$j$ randomly following
$P\left(i, j, t_{m}\right)= \begin{cases}\frac{F\left(\tau\left(i, j, t_{m}\right)\right)}{\sum_{(i, l) \in D_{i}} F\left(\tau\left(i, l, t_{m}\right)\right)} & \text { if }(i, j) \in D_{i} \\ 0, & \text { otherwise }\end{cases}$

Where $P\left(i, j, t_{m}\right)$ is the probability selected by an artificial ant at iteration $m$, from the source node $i$ to the target node (next node) $j$ along the connection $(i, j)$, $t_{m}$ is a strictly increasing function of $m$ and corresponds to the starting instant at iteration $m$, and when $m \rightarrow \infty, t_{m} \rightarrow \infty$ (For example, we can choose $t_{m}=\beta \cdot m$ for a small constant $\beta>0)$. $F(\tau)$ denotes a strictly increasing function with one real variable $\tau$, i.e. $d F / d \tau>0$ and $F(\tau)>0$, $\tau\left(i, j, t_{m}\right)$ is the amount of pheromone on the connection $(i, j)$ at iteration $m$, and $D_{i}$ is the set if and only if the source node is $i$ and the target node belongs to the feasible components. If the set $D_{i}$ is empty at some point in the solution construction, the ant is dropped and its solution construction is terminated.

In PHEROMONE UPDATE of GACO ALGORITHM described bellow, once all the ants have terminated their procedure described by ANT SOLUTION CONSTRUCTION of GACO ALGORITHM mentioned above, a pheromone update phase is started in which the pheromone trails are modified, the detail will be discussed in the following.

Let $\hat{s}$ be the best feasible solution found so far and $s_{t_{m}}$ be the best feasible solution in the current algorithm iteration $t_{m} ; f(\hat{s})$ and $f\left(s_{t_{m}}\right)$ are the corresponding objec-

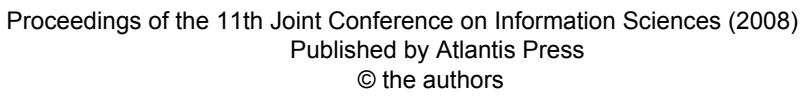


tive function (cost) values. The goal of the minimization problem is to find the globally optimal solution, i.e., a feasible candidate solution of minimum cost. The pheromone update procedure is described as follows.

PHEROMONE UPDATE Of GACO ALGORITHM

$$
\begin{aligned}
& \forall(i, j): \tau\left(i, j, t_{m}\right) \leftarrow \delta\left(t_{m}\right) \cdot \tau\left(i, j, t_{m-1}\right) \\
& \text { If } f\left(s_{t_{m}}\right)<f(\hat{s}), \text { then } \hat{s} \leftarrow s_{t_{m}} \\
& \forall(i, j) \in \hat{s}: \tau\left(i, j, t_{m}\right) \leftarrow \tau\left(i, j, t_{m}\right)+\mu\left(t_{m}\right)
\end{aligned}
$$

Where $t$ denotes time, $\delta(t)$ and $\mu(t)$ are continuous function of time $t$, we call $\delta(t)$ evaporation rate function and $\mu(t)$ global increasing function. Although $\delta(t)$ and $\mu(t)$ are continuous functions of time, only those discrete values $\delta\left(t_{m}\right)$ and $\mu\left(t_{m}\right) \quad(m=0,1,2, \cdots)$ corresponding to the discrete time $t=\left\{t_{m} \mid m=0,1, \cdots\right\}$ (where $\left.0 \leq t_{0}<t_{1}<\cdots<t_{m}<\cdots\right)$ are introduced in our PHEROMONE UPDATE of GACO ALGORITHM, which is convenient for computer simulation. The mathematical modeling for $\delta(t)$ and $\mu(t)$ is described as follows.

Obviously, the value of the pheromone can not be negative and will be decreased by the behavior of evaporation on all the connections, we have that $0<\delta(t)<1$. With the passage of time, more amount of pheromone will be evaporated. Therefore, we choose a strictly decreasing function $\delta(t)$ for describing pheromone evaporation, i.e. $d \delta(t) / d t<0$.

From the first expression $(\tau(i, j, t) \leftarrow$ $\delta(t) \cdot \tau(i, j, t)$ ) of PHEROMONE UPDATE of GACO ALGORITHM, we understand that if the value of $\delta(t)$ is close to 0 , then the updated pheromone $\tau(i, j, t)$ is also close to 0 , therefore, the changing rate of $\tau(i, j, t)$ is close to 0 , which may imply that $d \delta(t) / d t$ is proportional to $\delta(t)$ for modeling of $\delta(t)$. It corresponds to the case that there is no or very litter amount of pheromone on the connection $(i, j)$, or the amount of pheromone on the edge $(i, j)$ has been evaporated for a long time.

On the other hand, if the value of $\delta(t)$ is close to 1 , the updated pheromone $\tau(i, j, t)$ almost keeps unchanged by using the pheromone update procedure rule $\tau(i, j, t) \leftarrow \delta(t) \cdot \tau(i, j, t)$, or we say that the changing rate of $\tau(i, j, t)$ is also close to 0 , which may imply that $d \delta(t) / d t$ is proportional to $1-\delta(t)$ for modeling of $\delta(t)$. It corresponds to the state that the amount of pheromone on the connection $(i, j)$ maintains equilibrium between the procedure of the amount of pheromone trails decreasing by evaporation and increasing by the ants passed by.

As mentioned above, for evaporation rate function $\delta(t)$, a reasonable assumption is that $d \delta(t) / d t$ is proportional to $\delta(t)$ and $1-\delta(t)$, i.e.

$$
\frac{d \delta}{d t}=-\lambda \delta(1-\delta)
$$

where $\lambda$ is a constant and $\lambda>0$. The solution to differential equation (2) is

$$
\delta(t)=\frac{1}{1+C e^{\lambda t}}
$$

where $C$ is a constant. Let $\delta(0)=\delta_{0}$, we have

$$
\delta(t)=\frac{\gamma_{0} e^{-\lambda t}}{1+\gamma_{0} e^{-\lambda t}}
$$

where $\gamma_{0}=\delta_{0} /\left(1-\delta_{0}\right)$. If $\delta_{0} \in(0,1)$, then $\gamma_{0}>0$, from (4) we see that, in such a situation, the following holds: $\delta(t)>0$, $\inf \delta(t)=0$ and $\sup \delta(t)=1$. 
Now, we discuss the modeling of global increasing function $\mu(t)$.

From the update rule $\tau(i, j, t) \leftarrow$ $\tau(i, j, t)+\mu(t)$ and the concept of positive feedback, the positive function $\mu(t)$ can be added to those edges that belong to the best-so-far solution (including the solution in the current iteration). As the value of $\tau(i, j, t)$ is always finite, the upper bound of $\mu(t)$ must be finite. The key difference between $\mu(t)$ and $\delta(t)$ is that $\mu(t)$ should be a class of strictly increasing function with positive lower and upper bound, i.e. $0<A<\mu(t)<B<+\infty$, and $d \mu(t) / d t>0$.

Similar to the procedure of modeling of $\delta(t)$, we suppose that $d \mu(t) / d t$ is proportional to $\mu(t)-A$ and $B-\mu(t)$, i.e.

$$
\frac{d \mu}{d t}=\kappa(\mu-A)(B-\mu)
$$

where $\kappa$ is a positive constant. Then the general solution to differential equation (5) is described in the following

$$
\mu(t)=\frac{A-B C e^{\kappa(B-A) t}}{1-C e^{\kappa(B-A) t}}
$$

where $C$ is a constant. If we let $\mu(0)=\mu_{0}$, the solution to differential equation (5) is

$$
\mu(t)=\frac{A+B \eta_{0} e^{\kappa(B-A) t}}{1+\eta_{0} e^{\kappa(B-A) t}}
$$

where $\eta_{0}=\left(\mu_{0}-A\right) /\left(B-\mu_{0}\right)$. Clearly, if $\mu_{0} \in(A, B)$, then $\eta_{0}>0$, from (7) we have that $\mu(t)>0, A=\inf \mu(t)$ and $B=\sup \mu(t)$.

\section{Convergence Analysis for GACO Algorithm}

Proposition: Assume $0<\delta(t)<1$, $d \delta(t) / d t<0 \quad, \quad 0<A<\mu(t)<B<+\infty \quad$ and $d \mu(t) / d t>0$, if $\tau\left(i, j, t_{0}\right)>0$, then, for any $t_{m}>t_{0}$, the following holds:

$$
0<\tau\left(i, j, t_{m}\right)<\tau\left(i, j, t_{0}\right)+\frac{B}{1-\delta_{0}}
$$

where $B=\sup \mu(t), \delta_{0}=\delta(0)$.

Proof: The maximum possible amount of pheromone added to any edge $(i, j)$ after any iteration is $B$. Clearly, at iteration $t_{1}$, the maximum possible pheromone trail is

$$
\begin{aligned}
\tau\left(i, j, t_{1}\right) & =\delta\left(t_{1}\right) \cdot \tau\left(i, j, t_{0}\right)+\underset{(i, j)}{\operatorname{sgn}}(\hat{s}) \cdot \mu\left(t_{1}\right) \\
& <\delta\left(t_{0}\right) \cdot \tau\left(i, j, t_{0}\right)+B
\end{aligned}
$$

Where

$$
\underset{(i, j)}{\operatorname{sgn}(\hat{s})}= \begin{cases}1, & \text { if }(i, j) \in \hat{s} \\ 0, & \text { otherwise }\end{cases}
$$

Using $0<\delta(t)<1$ and $\tau\left(i, j, t_{0}\right)>0$, we get:

$$
\begin{aligned}
0 & <\delta\left(t_{1}\right) \cdot \tau\left(i, j, t_{0}\right) \leq \tau\left(i, j, t_{1}\right) \\
& <\delta\left(t_{0}\right) \tau\left(i, j, t_{0}\right)+B \sum_{m=0}^{\infty} \delta^{m}\left(t_{0}\right) \\
& <\tau\left(i, j, t_{0}\right)+\frac{B}{1-\delta\left(t_{0}\right)}
\end{aligned}
$$

Similarly, using (9), at iteration $t_{2}$, the maximum possible pheromone trail is

$$
\begin{aligned}
& \tau\left(i, j, t_{2}\right)=\delta\left(t_{2}\right) \tau\left(i, j, t_{1}\right)+\underset{(i, j)}{\operatorname{sgn}}(\hat{s}) \mu\left(t_{2}\right) \\
& <\delta^{2}\left(t_{0}\right) \tau\left(i, j, t_{0}\right)+\delta\left(t_{0}\right) B+B \\
& <\tau\left(i, j, t_{0}\right)+B \sum_{m=0}^{\infty} \delta^{m}\left(t_{0}\right) \\
& =\tau\left(i, j, t_{0}\right)+\frac{B}{1-\delta\left(t_{0}\right)}
\end{aligned}
$$


i.e.

$$
\begin{aligned}
& 0<\delta\left(t_{2}\right) \tau\left(i, j, t_{1}\right)<\tau\left(i, j, t_{2}\right) \\
&<\tau\left(i, j, t_{0}\right)+\frac{B}{1-\delta\left(t_{0}\right)}
\end{aligned}
$$

Obviously, the lower bound of $\tau\left(i, j, t_{m}\right)$ is: $\tau\left(i, j, t_{m}\right) \geq \delta\left(t_{m}\right) \tau\left(i, j, t_{m-1}\right) \geq \delta\left(t_{m}\right) \delta\left(t_{m-1}\right) \tau\left(i, j, t_{m-2}\right)$

$$
\geq \cdots \geq \prod_{k=1}^{m} \delta\left(t_{k}\right) \tau\left(i, j, t_{0}\right)>0
$$

As $t_{0}<t_{1}<\cdots<t_{m-1}<t_{m}$ and $\delta\left(t_{0}\right)>\delta\left(t_{1}\right)>$ $\cdots>\delta\left(t_{m-1}\right)>\delta\left(t_{m}\right)$, the upper bound of the maximum amount of pheromone for any edge $(i, j)$ at any iteration $m$ is

$$
\begin{aligned}
\tau\left(i, j, t_{m}\right) & =\delta\left(t_{m}\right) \tau\left(i, j, t_{m-1}\right)+\underset{(i, j)}{\operatorname{sgn}}(\hat{s}) \mu\left(t_{m}\right) \\
& <\delta\left(t_{m}\right) \tau\left(i, j, t_{m-1}\right)+B \\
& <\delta\left(t_{m}\right) \delta\left(t_{m-1}\right) \tau\left(i, j, t_{m-2}\right)+\delta\left(t_{m}\right) B+B \\
& <\delta^{2}\left(t_{0}\right) \tau\left(i, j, t_{m-2}\right)+\delta\left(t_{0}\right) B+B \\
& <\cdots<\delta^{m}\left(t_{0}\right) \tau\left(i, j, t_{0}\right)+B \sum_{i=1}^{m} \delta\left(t_{0}\right)^{m-i} \\
& <\tau\left(i, j, t_{0}\right)+\frac{B}{1-\delta\left(t_{0}\right)}
\end{aligned}
$$

Therefore, for any $t_{m}>t_{0}$, the following holds:

$$
0<\tau\left(i, j, t_{m}\right)<\tau\left(i, j, t_{0}\right)+\frac{B}{1-\delta\left(t_{0}\right)}
$$

For convenient discussion in the following, $\tau_{\text {lowb }}$ and $\tau_{\text {upb }}$ are introduced to denote the lower bound and the upper bound of pheromone trails for any edge $(i, j)$ respectively, i.e. $\tau_{\text {lowb }}=0$ and $\tau_{\text {upb }}=\tau\left(i, j, t_{0}\right)+B /\left(1-\delta\left(t_{0}\right)\right)$.

The algorithm is initialized as follows. First, Generate a feasible solution $\hat{s}$, and then, $\forall(i, j)$, set $\tau\left(i, j, t_{0}\right)=\tau_{0}$, where $\tau_{\text {lowb }}<\tau_{0}<\tau_{\text {upb }}$, is a parameter. If $t_{0}=0$ corresponds to the initial state, then the upper bound $\tau_{\text {upb }}$ can be given as $\tau_{\text {upb }}=\tau_{0}+B /\left(1-\delta_{0}\right)$.

Theorem: Let $P\left(t_{m}\right)$ be the probability that GACO algorithm finds the globally optimal solution at least once within the first $m$ iterations, the following holds:

$$
\lim _{m \rightarrow \infty} P\left(t_{m}\right)=1
$$

Proof: Due to $\tau \in\left[\tau_{\min }, \tau_{\max }\right]$ $\subset\left(\tau_{\text {lowb }}, \tau_{\text {upb }}\right)$, we can construct a class of positive function $F(\tau)>0$, which is strictly increasing on $\left[\tau_{\text {lowb }}, \tau_{\text {upb }}\right] \supset$ $\left[\tau_{\min }, \tau_{\max }\right]$, i.e. $d F / d \tau>0$. Because $\tau_{\min }>0$ and $\tau_{\max }<\tau_{\text {upb }}$, we can guarantee that any feasible choice in (1) is done with a probability $p_{\min }>0$, and that the lower bound for $p_{\min }$ can be given as

$$
\begin{aligned}
p_{\min } & =\frac{F_{\min }(\tau)}{\left(N_{c}-1\right) \cdot F_{\max }(\tau)+F_{\min }(\tau)} \\
& =\frac{F\left(\tau_{\min }\right)}{\left(N_{c}-1\right) \cdot F\left(\tau_{\max }\right)+F\left(\tau_{\min }\right)}
\end{aligned}
$$

where $N_{c}$ is the cardinal number of the set for the considered problem. Then, the globally optimal solution can be generated with a probability $p \geq p_{\min }^{n}>0$ (where $n<+\infty$ is the maximum length of a sequence associated with the considered problem), therefore, the probability that GACO algorithm does not find the globally optimal solution within the first $m$ iterations is $(1-p)^{m}$. Because it is enough that one ant finds the globally optimal solution, the probability that GACO algorithm finds the globally optimal solution at least once within the first $m$ iterations can be given as

$$
P\left(t_{m}\right)=1-(1-p)^{m}
$$


Clearly, as $0<p<1$, we have that $\lim _{m \rightarrow \infty} P\left(t_{m}\right)=\lim _{t \rightarrow \infty} P(t)=1$. Therefore, for an arbitrary choice of a small $\varepsilon>0$ and for a sufficiently large time $t$, it holds that $P(t)>1-\varepsilon$.

\section{Conclusions}

In GACO algorithm, the general form of function $F(\tau)$ is a strictly increasing function with variable $\tau$, i.e. $F^{\prime}(\tau)>0$, which is different from the formula presented in [7]. In [7], the polynomial function $\tau^{\alpha}$ was introduced, where $\alpha>0$, which could be traced back to earlier literature [8], a more special form of function $\tau^{2}$ proposed by S. Goss et al. Obviously, $\tau^{\alpha}(\alpha>0)$ is only a special form of function $F(\tau)$. The improvement proposed in this paper may get more general form of convenient expression for some applications.

In GACO algorithm, a new feasible pheromone trails update method is introduced for mathematical modeling of $\delta(t)$ and $\mu(t)$, which is different from the evaporation factor $1-\rho$ and a function $g(s)$ proposed in $\mathrm{ACO}_{\mathrm{gb}, \tau_{\text {min }}}$ [7]. On the other hand, for the pheromone update rule, a lower limit $\tau_{\min }$ on the range of feasible pheromone trails enforced by $\mathrm{ACO}_{\mathrm{gb}, \tau_{\min }}$ is not required in GACO algorithm.

The important convergence theorem shows that, the probability of finding the globally optimal solution at least once is $P(t)>1-\varepsilon$. On the point of view in theory, the important convergence property tells us that, at least to some extent, GACO algorithm can be used to some combinatorial optimization problems.
[1] M. Dorigo, E. Bonabeau, and G. Theraulaz, "Ant algorithms and stigmergy,” Future Gener. Comput. Syst., vol. 16, no. 8, pp. 851-871, 2000.

[2] M. Dorigo and G. Di Caro, "The ant colony optimization meta-heuristic," in New Ideas in Optimization, D. Corne, M. Dorigo, and F. Glover, Eds. London, U.K.: McGraw-Hill, pp. 1132, 1999.

[3] M. Dorigo, G. Di Caro, and L. M. Gambardella, “Ant algorithms for discrete optimization,” Artif. Life, vol. 5, no. 2, pp. 137-172, 1999.

[4] M. Birattari, G. Di Caro, and M. Dorigo, "For a formal foundation of the ant programming approach to combinatorial optimization. Part 1: The problem, the representation, and the general solution strategy," ATR Human Information Processing Laboratories, Kyoto, Japan, Tech Rep. TR-H-301, Dec. 2000.

[5] N. Meuleau and M. Dorigo, “Ant colony optimization and stochastic gradient descent," Artif. Life, vol. 8, no. 2, pp. 103-121, 2002.

[6] , "A graph-based ant system and its convergence,” Future Gener. Comput. Syst., vol. 16, no. 8, pp. 873-888, 2000.

[7] T. Stützle and M. Dorigo, “A Short Convergence Proof for a Class of Ant Colony Optimization Algorithms," IEEE Trans. Evolutionary Computation, vol.6, no.4, pp.358-365, 2002.

[8] S. Goss, S. Aron, J. L. Deneubourg, and J. M. Pasteels, "Self-organized Shortcuts in the Argentina Ant," Naturwissenschaften, 76, pp.579-581, 1989.

\title{
5. References
}

\author{
Proceedings of the 11th Joint Conference on Information Sciences (2008) \\ Published by Atlantis Press \\ (C) the authors
}

